\title{
Enhanced myeloid differentiation factor 88 promotes tumor metastasis via induction of epithelial-mesenchymal transition in human hepatocellular carcinoma
}

\author{
R-J Jia ${ }^{1,2,8}$, L Cao ${ }^{1,3,8}$, L Zhang ${ }^{1,8}$, W Jing ${ }^{4}$, R Chen ${ }^{1}$, M-H Zhu ${ }^{4}$, S-W Guo ${ }^{4}$, G-B Wu ${ }^{5}$, X-Y Fan ${ }^{1}$, H Wang ${ }^{1}$, Y-Y Zhang ${ }^{3}$, X-Y Zhou ${ }^{*, 4}$, \\ J Zhao ${ }^{*, 1,2,6}$ and Y-J Guo ${ }^{1,2,6,7}$
}

Metastasis is the leading cause of death in patients with hepatocellular carcinoma (HCC) after curative resection. Therefore, it is critical to understand the mechanisms underlying tumor metastasis in HCC. We have previously shown that elevated expression of myeloid differentiation factor 88 (MyD88) may promote tumor growth and metastasis in HCC. In this study, we reported that enhanced expression of MyD88 promoted epithelial-mesenchymal transition (EMT) properties and tumor-initiating capabilities in HCC cells. MyD88 was found to be able to interact with p85, a regulatory subunit of phosphoinositide 3-kinase (PI3-K), independent of TLR/LL-1Rmediated response and caused PI3-K/v-akt murine thymoma viral oncogene homolog (Akt) activation, which resulted in subsequent phosphorylation of glycogen synthase kinase-3 $\beta$ and stabilization of Snail, a critical EMT mediator. Consistently, we observed a significant correlation between MyD88 expression and p-Akt levels in a cohort of HCC patients, and found that the combination of these two parameters have better prognostic value for HCC patients. Taken together, these results suggest that elevated MyD88 may facilitate HCC metastasis by promoting EMT properties and tumor-initiating capabilities via PI3-K/Akt pathway.

Cell Death and Disease (2014) 5, e1103; doi:10.1038/cddis.2014.71; published online 6 March 2014

Subject Category: Cancer

The inflammatory response that is considered to be the most important environmental factor contributing to tumor progression by promoting proliferation, antiapoptosis, invasion, and angiogenesis ${ }^{1-3}$ can be initiated by several types of patternrecognition receptors. The Toll-like receptors (TLRs) are the best-characterized ones. ${ }^{4}$ TLR/IL-1R-mediated inflammatory response relies on the adapter proteins containing TIR domain to bridge receptors and corresponding intracellular molecules to transduce signals. Among such molecules, the first identified one is the myeloid differentiation factor 88 (MyD88). MyD88 is used by all known TLRs except TLR3, and it also serves as a signaling adapter for IL-1R and IL-18R.

Several studies have demonstrated the role of MyD88 in the protumorigenic inflammatory response..$^{5-7}$ In addition to its role in inflammation-associated tumorigenesis, MyD88 may act intrinsically in epithelial cells to promote carcinogenesis via non-inflammatory functions. MyD88 was able to promote the development of MCA-induced fibrosarcomas, a model that has not been classically defined as having a significant inflammatory origin. ${ }^{7}$ The non-inflammatory function of MyD88 for carcinogenesis in mice in vivo was elucidated in a RAS-dependent skin carcinogenesis model. Directly interacting with activated Erk, MyD88 was found to have a crucial role in RAS signaling, cell-cycle control, and cell transformation. $^{8}$

Indeed, abnormal expression of MyD88 has been found in various types of cancer and is related to tumor development. In colorectal cancer (CRC), high expression of MyD88 was frequently detected in CRC with liver metastasis. ${ }^{9}$ SKOV3 cells, an ovarian cancer cell line obtained from the ascites of a patient with advanced, metastatic ovarian cancer, expressed high level of MyD88. ${ }^{10}$ Ovarian cancer patients whose tumors did not express MyD88 improved progression-free interval compared with patients whose tumors expressed MyD88,

\footnotetext{
${ }^{1}$ International Joint Cancer Institute, The Second Military Medical University, 800 Xiangyin Road, Shanghai 200433, People's Republic of China; ${ }^{2}$ School of Pharmacy, Liaocheng University, 1 Hunan Road, Liaocheng 252059, People's Republic of China; ${ }^{3}$ School of Medicine, Shanghai Jiao Tong University, 227 South Chongqing Road, Shanghai 200025, People's Republic of China; ${ }^{4}$ Changhai Hospital, The Second Military Medical University, Shanghai 200433, People's Republic of China; ${ }^{5}$ Guangxi Cancer Hospital, Guangxi Medical University, Nanning 530021, People's Republic of China; ${ }^{6}$ PLA General Hospital Cancer Center, PLA postgraduate School of Medicine, 28 Fuxing Road, Beijing 100853, People's Republic of China and ${ }^{7}$ National Engineering Research Center of Antibody Medicine and State Key Laboratory of Antibody Medicine and Targeting Therapy, 99 Libing Road, Shanghai 201203, People's Republic of China

*Corresponding author: X-Y Zhou, Changhai Hospital, The Second Military Medical University, Shanghai 200433, People's Republic of China. Tel: + 862131161627 ; Fax: + 8621 65306667; E-mail: xuyu2006@medmail.com.cn

or J Zhao, International Joint Cancer Institute, The Second Military Medical University, 800 Xiangyin Road, New Building 9-11th Floor, Shanghai 200433, People's Republic of China. Tel: + 8621 81870807; Fax: + 8621 81870801; E-mail: zhaojian@smmu.edu.cn

${ }^{8}$ These authors contributed equally to this work.

Keywords: tumor metastasis; myeloid differentiation factor 88; epithelial-mesenchymal transition

Abbreviations: HCC, hepatocellular carcinoma; EMT, epithelial-mesenchymal transition; MyD88, myeloid differentiation factor 88; PI3-K, phosphoinositide 3-kinase; Akt, v-akt murine thymoma viral oncogene homolog; GSK-3 $\beta$, glycogen synthase kinase-3 $\beta$

Received 16.12.13; revised 27.1.14; accepted 28.1.14; Edited by A Stephanou
} 
which was statistically significant. ${ }^{11}$ The expression of MyD88 was significantly higher in the 10 cases of hepatocellular carcinoma (HCC) with portal vein tumor thrombi than that in the metastasis-free HCCs at the time of surgery. ${ }^{12}$ In our previous study, ${ }^{13}$ we reported that MyD88 was frequently upregulated in HCCs, which was closely related with the worse stage of tumor and the higher recurrent rate in $\mathrm{HCC}$ patients. Ectopic expression of MyD88 promoted HCC cell proliferation and invasion in vitro. Suppression of MyD88 expression with lentivirus-encoding shRNA reduced tumor growth and invasion in vitro, as well as lung metastasis in vivo. ${ }^{13}$ Our findings, and previous observations, strongly indicate that elevated MyD88 is involved in tumor metastasis and that it may serve as a prognostic factor for cancer patients.

Epithelial-mesenchymal transition (EMT) is defined as the process in which epithelial cells lose their epithelial signatures while acquiring the characteristics of mesenchymal cells including morphology, cellular structure, and biological function. ${ }^{14}$ EMT usually occurs in the critical phases of embryonic development. However, this developmental program also has an important role in tumor metastasis. The EMT cells also acquire stem cell-like properties, including the ability of self-renewal and the ability to resist apoptotic stimulations, which is critical for the survival and metastasis of disseminated cancer cells. ${ }^{15-17}$ Induction of EMT in immortalized human mammary epithelial cells was reported to result in the expression of stem cell markers and increased ability associated with stemness. On the other hand, stem cell-like cells express EMT markers. ${ }^{16}$ These findings illustrate a direct link between the EMT and the acquisition of epithelial stem cell properties.

Characteristic downregulation of E-cadherin is regarded as the key step of EMT. Transcription factors that regulate E-cadherin expression include zinc-finger transcriptional repressors (Snail, Slug, Zeb1, and Zeb2) and bHLH family (Twist). ${ }^{18,19}$ Transforming growth factor- $\beta$, the main and the best-characterized upstream inducers of EMT, may function through multiple distinct signaling pathways. ${ }^{15,20}$ Moreover, a variety of other cancer-associated cascades have emerged as important regulatory signalings for EMT, which include phosphoinositide 3-kinase (PI3-K)/v-akt murine thymoma viral oncogene homolog (Akt)-, Wnt-, Notch-, Hedgehog- or $\mathrm{NF}-\kappa \mathrm{B}$-dependent pathways. ${ }^{21}$ Some of these pathways can act in concert to trigger EMT.

In this study, we demonstrated that elevated MyD88 is able to induce properties of EMT and tumor-initiating cells via PI3$\mathrm{K} /$ Akt/glycogen synthase kinase-3 $\beta$ (GSK-3 $\beta$ )/Snail pathway to facilitate tumor metastasis in HCC.

\section{Results}

MyD88 induces characteristics of EMT in hepatoma cells. Our previous study demonstrated that elevated MyD88 was able to promote tumor growth and metastasis, and may serve as a biomarker for prognosis in patients with HCC. ${ }^{13}$ To elucidate whether MyD88 promotes tumor metastasis via induction of EMT, a process by which tumor-associated epithelial cells obtain mesenchymal features, ${ }^{22}$ we employed lentivirus-encoding MyD88 cDNA to overexpress MyD88 in PLC/PRF/5 with low endogenous MyD88 and Hep3B with medium endogenous MyD88, or lentivirus-encoding shRNA to knockdown MyD88 in Hep3B and HCC-LM3, which had medium level of endogenous MyD88 (Supplementary Figures 1a and b). Accompanied with enhanced migration and invasion abilities (Supplementary Figures 2a-e), enforced MyD88 expression resulted in the loss of epithelial maker (E-cadherin) and the gain of mesenchymal markers (vimentin and $\mathrm{N}$-cadherin) in PLC/PRF/5 and Hep3B cells (Figure 1a). The mRNA levels of EMT-promoting transcription factors like Snail, Slug, Zeb1, and Zeb2 were increased when MyD88 expression was upregulated (Figure 1b). In contrast, silencing of MyD88 caused enhanced E-cadherin expression and reduced vimentin and $\mathrm{N}$-cadherin expression, together with reduced expression of EMT-promoting transcription factors in Hep3B as well as HCC-LM3 cells (Figures 1c and d). Following overexpression of MyD88, PLC/PRF/5 cells showed spindle-like, fibroblastic morphology, one of the main characteristics of EMT, whereas more epithelial morphology was observed in MyD88-silenced Hep3B cells (Figure 1e). Immunofluorescent staining showed reduced membranous staining of E-cadherin and increased vimentin and $\mathrm{N}$-cadherin staining in MyD88overexpressed PLC/PRF/5 cells, whereas enhanced epithelial cell-like staining was observed in MyD88-silenced Hep3B cells (Figure 1e).

We have examined the expression of MyD88 in 110 cases of HCC previously. ${ }^{13}$ Here, we assessed the expression of E-cadherin in these specimens. In the tumor tissues, $45.5 \%$ (50/110) had membranous staining of E-cadherin, which was considered as normal, and 54.5\% (60/110) had aberrant E-cadherin expression with cytoplasmic staining or negative staining. The statistical analysis revealed a strong negative correlation between MyD88 expression and E-cadherin membranous expression ( $P=0.000, r=-0.383$, Figure 1f). These results suggest that the enhanced expression of MyD88 may induce EMT in HCC cells.

MyD88 enhances tumor-initiating capacity in hepatoma cells. As EMT is able to confer cells with cancer stem cell-like properties, ${ }^{16}$ we therefore screened changes in mRNA levels of genes related to self-renewal of stem cells. Forced expression of MyD88 greatly enhanced expressions of Nanog and Notch-1 in PLC/PRF/5 (Figure 2a), whereas silencing of MyD88 caused significant reduction of several critical genes required for self-renewal of stem cells including Oct-4, Nanog, Sox-2, Notch-1, and Hif-1 $\alpha$ in Hep3B (Figure 2a). Moreover, we evaluated the expression of several putative hepatic stem cell markers like CK19, EpCAM, and CD133. ${ }^{23-25}$ As shown in the lower panel of Figure $2 \mathrm{a}$, the expression of CK19 was greatly enhanced in MyD88-overexpressed PLC/PRF/5 cells, whereas the expression of CD133 and EpCAM did not change significantly. When MyD88 was knocked down, the mRNA levels of CK19 and CD133 were significantly decreased in Hep3B cells. Recently, liver 'cancer stem cells' have been identified by several cell surface molecules such as CD133, EpCAM, and CD90. ${ }^{24-26}$ Using flow cytometry analysis, we examined the expressions of these markers. As shown in Figure $2 \mathrm{~b}$, although CD90 expression was statistically unchanged, 

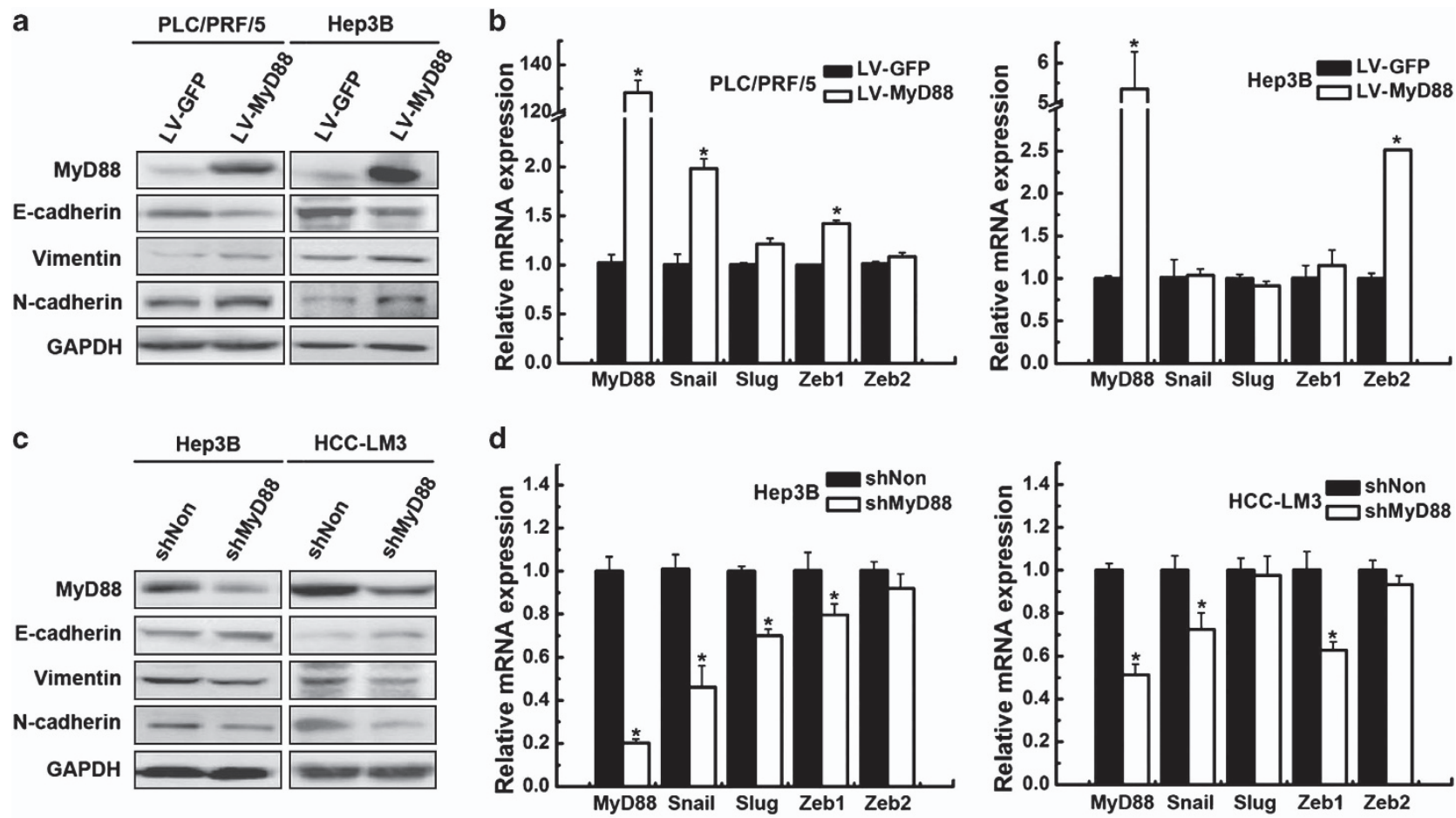

e

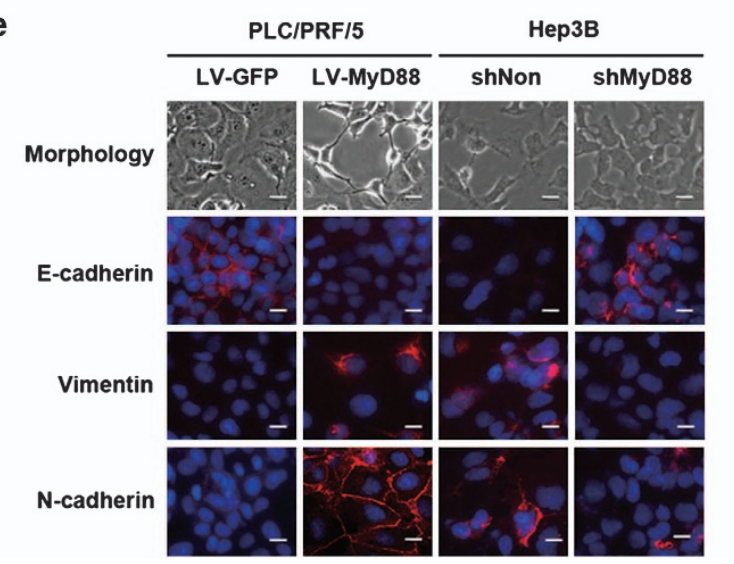

f

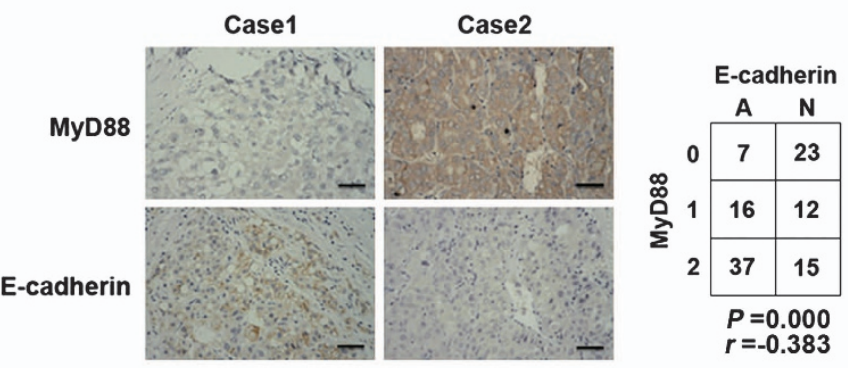

Figure 1 MyD88 induces characteristics of EMT in HCC cells. (a, c) Western blot analysis and (e) immunofluorescence staining showed decreased expression of epithelial marker (E)-cadherin) and upregulated expression of mesenchymal markers (vimentin and N-cadherin) when MyD88 was overexpressed. In contrast, the knockdown of MyD88 resulted in increased expression of epithelial marker and decreased expression of mesenchymal markers. Scale bar, $25 \mu \mathrm{m}$. (b, d) Fold-change in mRNA levels of several EMT-related transcription factors after MyD88 was overexpressed or knocked down. Fold-change of mRNA expression was calculated by normalization to the control as indicated in Materials and Methods. (f) Representative views of immunostaining of MyD88 and E-cadherin and statistical correlation between MyD88 and E-cadherin expression are shown. A: aberrant; N: normal. Data represent mean \pm S.D. * indicates $P<0.05$. Scale bar, $100 \mu \mathrm{m}$

the expressions of CD133 and EpCAM in Hep3B cells were decreased after MyD88 was knocked down. However, the expressions of these markers did not change significantly in PLC/PRF/5 cells when MyD88 was overexpressed (Supplementary Figure 3a). The side population (SP) cells, a small population of tumor cells, have many properties of stem cells. Lately, SP cells were used in an attempt to isolate a stem cell-like fraction in cancer cells. ${ }^{27}$ These SP cells were practically diminished in the presence of Hoechst 33342 and verapamil, a calcium channel blocker. Flow cytometry analysis with Hoechst 33342 staining demonstrated that after MyD88 was knocked down, the SP proportion in Hep3B cells declined to $0.51 \%$, compared with
$1.53 \%$ in the control group (Figure 2c). But when MyD88 was overexpressed in PLC/PRF/5 cells, the SP fraction did not alter significantly (Supplementary Figure $3 b$ ). To further analyze the expression of MyD88 in putative hepatic cancer stem cells, the fresh clinical specimens were collected.

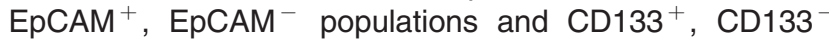
populations were isolated by magnetic bead cell sorting. $\mathrm{CD}_{133^{+}}$and $\mathrm{EpCAM}^{+}$populations displayed much higher mRNA level of MyD88 than that in CD133- and EpCAM ${ }^{-}$ populations, respectively (Figure $2 \mathrm{~d}$ ). We then investigated the impact of MyD88 on the tumor-initiating capability via sphere-forming experiment. In PLC/PRF/5 cells, there was a significant increase in the amount of hepatospheres when 


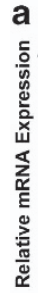

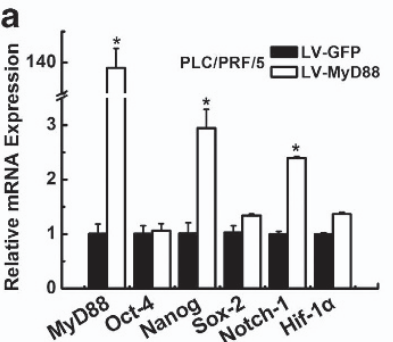

b
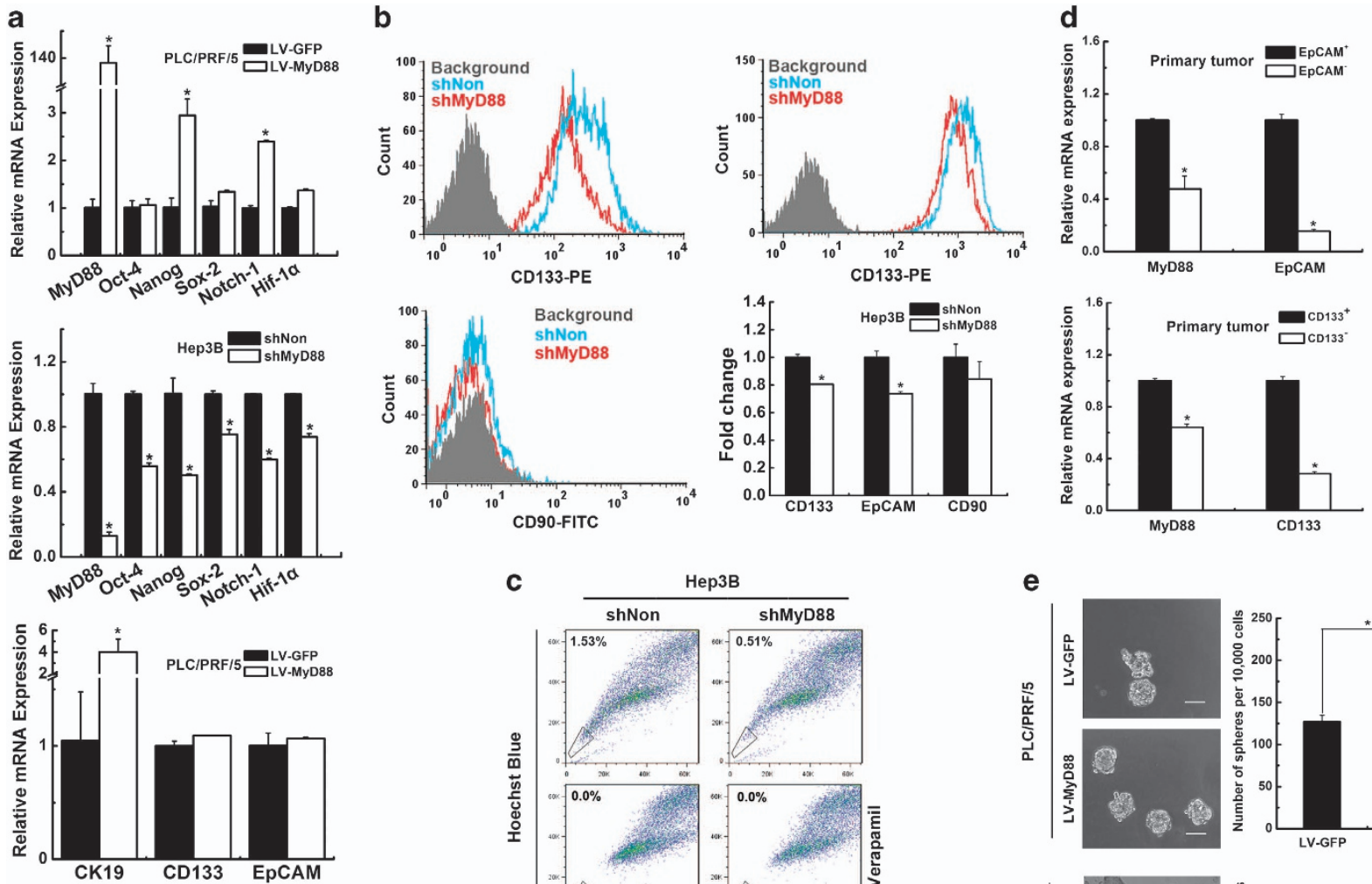

C
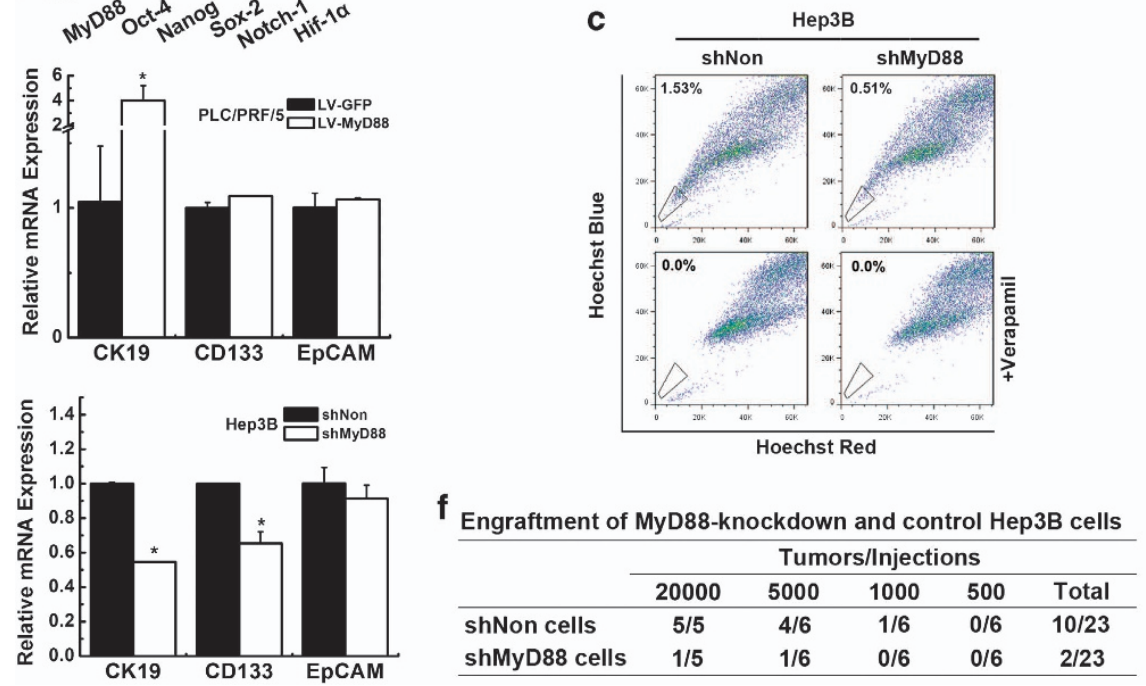

e
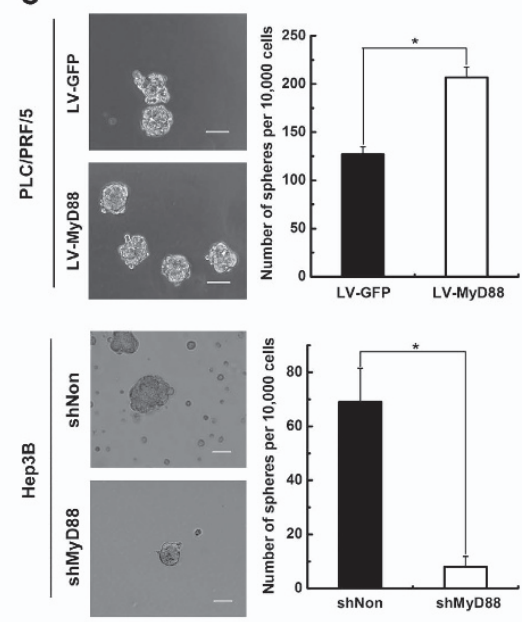

Figure 2 MyD88 enhances tumor-initiating capacity in HCC. (a) Fold-change in mRNA levels of several stemness-related genes and putative hepatic stem cell markers when MyD88 was overexpressed or knocked down. Fold-change in mRNA expression was calculated by normalization to the control as indicated in Materials and Methods. (b) Flow cytometer analysis of Hep3B cells stained with anti-CD133, anti-EpCAM, and anti-CD90 after MyD88 was knocked down. (c) The SP cell fraction was analyzed in

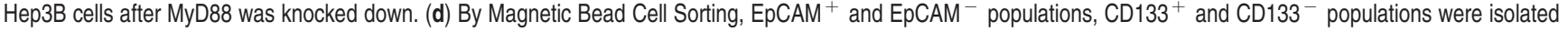
from fresh primary HCC samples, and the mRNA levels of MyD88, EpCAM, and CD133 were tested. (e) Representative images and quantification of the sphere formation in PLC/PRF/5 and Hep3B cells after MyD88 was overexpressed or knocked down, respectively. Scale bar, $100 \mu$ m. (f) Tumor incidences in SCID mice injected with the indicated number of shNon- or shMyD88-infected Hep3B cells after 6 weeks are shown. Data represent mean \pm S.D. ${ }^{*}$ indicates $P<0.05$

MyD88 was overexpressed (Figure 2e). In contrast, Hep3B cells formed much fewer and smaller spheres when MyD88 was knocked down (Figure 2e). To examine the effect of MyD88 on the tumorigenicity of hepatoma cells, a series of gradient PLC/PRF/5 and Hep3B cells were injected subcutaneously into severe combined immunodeficient (SCID) mice. As displayed in Supplementary Figure 3c, tumor incidence between LV-GFP group and LV-MyD88 group made little difference. But a notable difference in tumorigenicity was observed between shNon group and shMyD88 group 6 weeks after mice were inoculated with Hep3B cells (Figure 2f). All these results demonstrate that MyD88 is required to maintain tumor-initiating capacity in hepotoma cells.

MyD88 interacts with p85 to activate PI3-K/Akt and induce EMT. Accumulating studies have suggested that
PI3-K/Akt activation has a momentous role in induction of EMT during tumor progression. ${ }^{28-30}$ We have previously reported that downregulation of MyD88 was able to inhibit the intrinsic activation of Akt in HCC-LM3 cells with medium level of endogenous MyD88. ${ }^{13}$ Here, we found that the phosphorylation level of Akt was fairly increased in PLC/PRF/5 cells with enforced MyD88 expression and decreased in MyD88-silenced Hep3B cells (Figure 3a). Blockage of Akt by DN-Akt, a dominant negative mutant of Akt, dramatically attenuated MyD88-elicited EMT and invasive capability in PLC/PRF/5 cells (Figures $3 b$ and d). Nude mice inoculated with PLC/PRF/5/LV-MyD88 cells in spleen displayed more and larger xenografts in the liver in comparison with control mice. Treatment with Akt inhibitor, MK2206, significantly inhibited the xenograft formation in these mice (Figure 3e). 


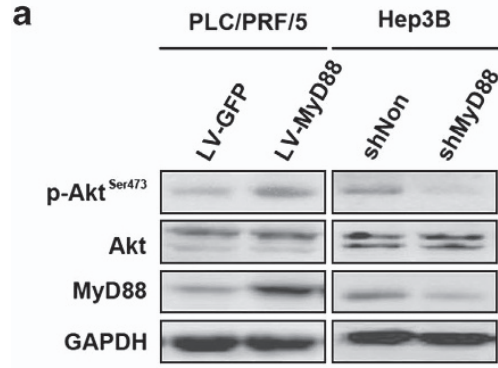

C

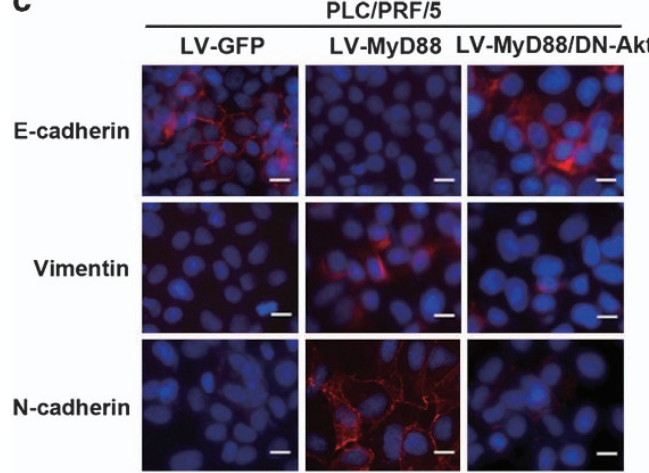

b

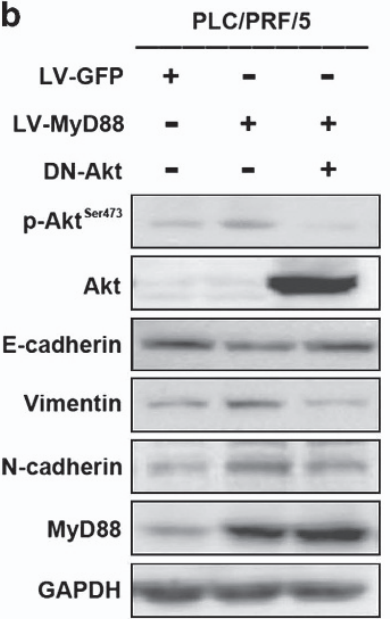

d
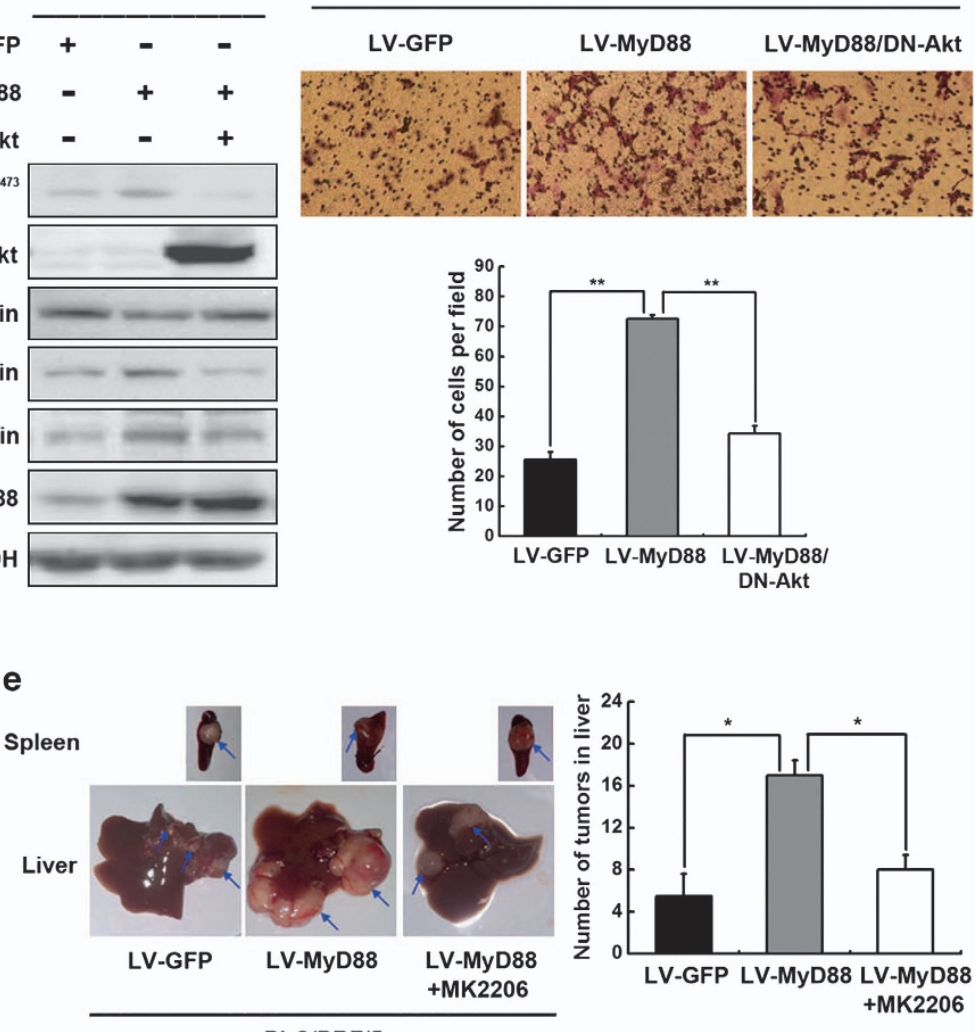

PLC/PRF/5

Figure 3 MyD88 activates Akt, promoting metastatic potential of HCC cells. (a) MyD88 was overexpressed or knocked down as indicated and the phosphorylation levels of Akt were analyzed by western blot assay. (b, $\mathbf{c}$ ) The expressions of epithelial marker (E-cadherin) and mesenchymal markers (vimentin and N-cadherin) were detected by western blot assay and immunofluorescence staining after PLC/PRF/5/LV-MyD88 cells were infected with DN-Akt. Scale bar, $25 \mu \mathrm{m}$. (d) PLC/PRF/5/LV-MyD88 cells were infected by DN-Akt and the invasive properties were analyzed by invasion assay using Matrigel-coated Corning chamber as described in Materials and Methods. (e) Nude mice were given intrasplenic injections of hepatoma cells and then intragastric administration of MK2206 (the inhibitor of Akt) as described in the Materials and Methods, and photographs of HCC tumors growth in the spleens (above) and livers (below) are shown. The number of tumors in each group $(n=4)$ five weeks after inoculation was counted. Data represent mean \pm S.D. ${ }^{*}$ indicates $P<0.05,{ }^{* *}$ indicates $P<0.01$

As acknowledged, Akt is chiefly activated by PI3-K. In order to assess the effect of MyD88 on PI3-K, we measured PI3-K activity using competitive ELISA. The results showed that in hepatoma cells, the presence of MyD88 significantly enhanced the activity of PI3-K and the absence of MyD88 greatly reduced the activity of PI3-K (Figure 4a). Furthermore, to test whether MyD88 could enhance PI3-K activity via a direct mechanism, we performed coimmunoprecipitation assays. The results revealed that enforced MyD88 expression enhanced the binding of MyD88 with p85, a regulatory subunit of PI3-K, in PLC/PRF/5 cells (Figure 4b), whereas silencing MyD88 was able to attenuate the association of MyD88 with p85 in Hep3B cells (Figure 4c). Ectopic expression of $\Delta p 85$, a dominant negative mutant of p85, greatly attenuated MyD88triggered Akt phosphorylation, further confirming that PI3-K was involved in MyD88-induced Akt activation (Figure 4d). Taken together, these findings suggest that MyD88 interacts with p85 to activate PI3-K/Akt and induce EMT.

MyD88 regulates EMT via Akt/GSK-3 $\beta /$ snail pathway. GSK-3 $\beta$ has been documented to be regulated by Akt and is involved in EMT through the regulation of Snail. ${ }^{31}$ To test whether GSK-3 $\beta$ is involved in MyD88-mediated EMT, we examined the effect of MyD88 on GSK-3 $\beta$ activation. As shown in Figure 5a, phosphorylation of GSK-3 $\beta$ was notably enhanced by MyD88 overexpression and dramatically decreased by MyD88 knockdown in hepatoma cells. As expected, the expression of Snail, which is a predominant mediator of EMT and tightly regulated by GSK-3 $\beta$ at protein level, was significantly increased by MyD88 overexpression and decreased by MyD88 knockdown (Figure 5a). Consistently, overexpression of Ser9A-GSK-3 $\beta$, a constitutively active mutant, reduced MyD88-triggered Snail induction (Figure 5b). Suppression of Akt activation by DN-Akt remarkably attenuated MyD88-elicited GSK-3 $\beta$ phosphorylation and Snail expression in hepatoma cells (Figure $5 \mathrm{c}$ ). Furthermore, knockdown of Snail by small interfering RNA (siRNA) targeting Snail greatly attenuated MyD88-triggered enhanced invasive ability in PLC/PRF/5 cells (Figures $5 d$ and e). All these results suggest that $A k t / G S K-3 \beta /$ Snail pathway is critical in MyD88-mediated EMT and metastasis in HCC cells.

Combination of MyD88 and p-Akt provides better prognostic value for HCC. Increasing evidence reveals that a combination of multiple biological markers might be more powerful than any single one for the estimation of 


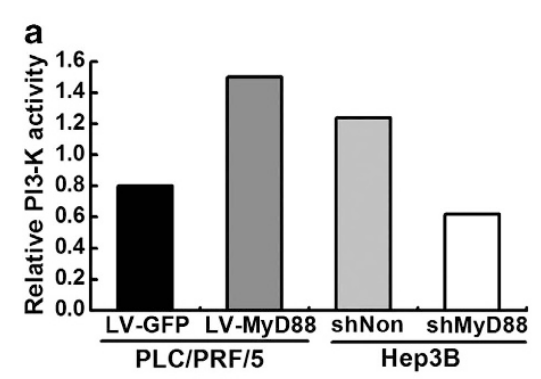

c

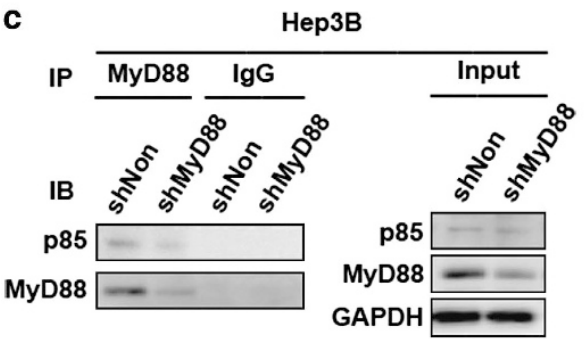

b

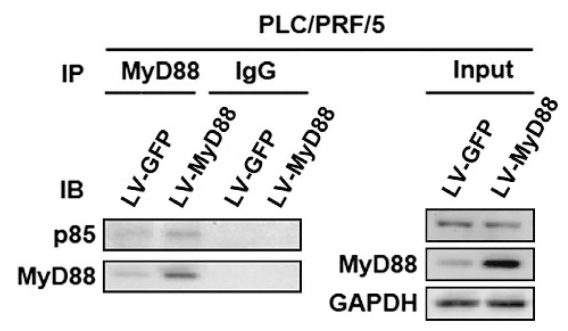

d

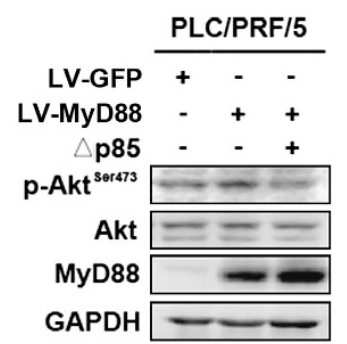

Figure 4 MyD88 associates with p85 to activate PI3-K/Akt signaling. (a) PI3-K was immunoprecipitated and its activity was detected as described in Materials and Methods. (b, c) After overexpression or knockdown of MyD88, the cell lysates were subjected to coimmunoprecipitation and western blot analysis. (d) PLC/PRF/5 cells stably expressing MyD88 were transfected with $\Delta \mathrm{p} 85$, and the phosphorylation level of Akt was analyzed by western blot assay

a

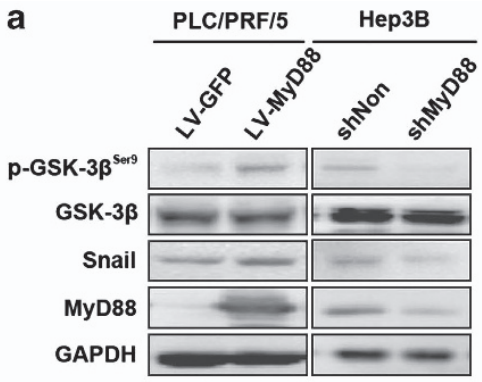

b

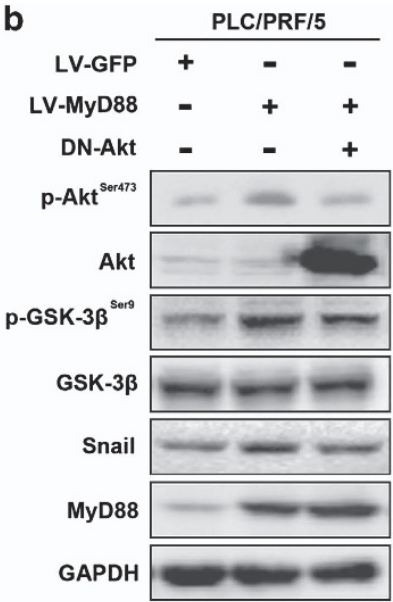

c

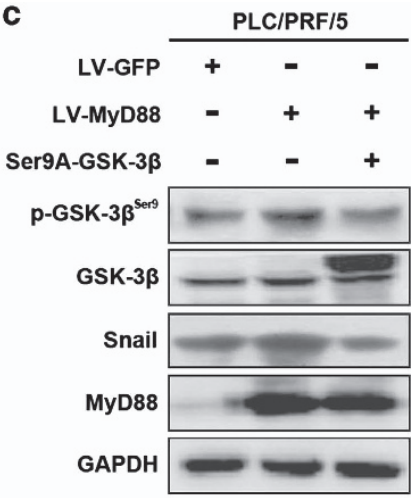

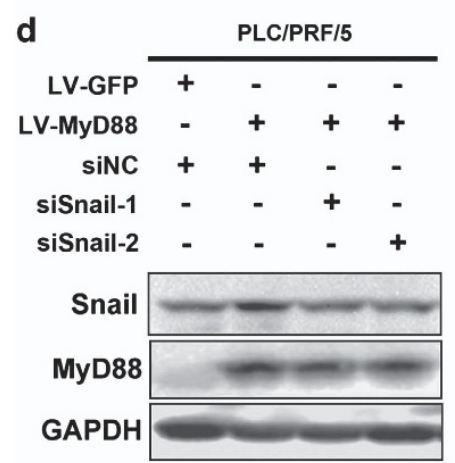

e

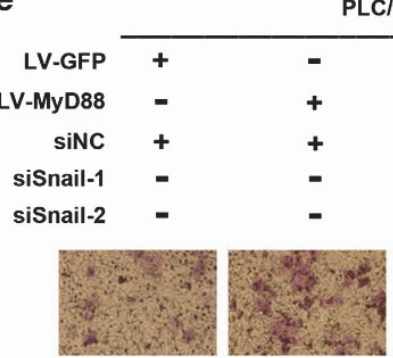

PLC/PRF/5

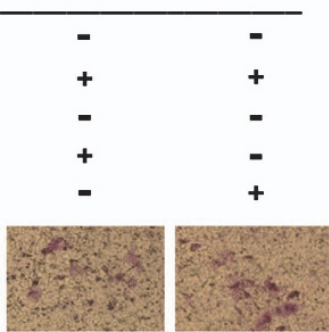

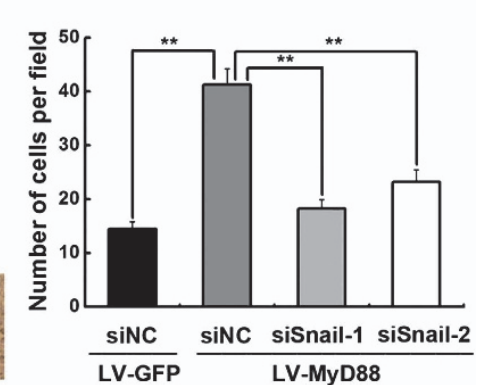

Figure 5 MyD88 regulates EMT via Akt/GSK-3 $\beta$ /Snail pathway. (a) MyD88 was overexpressed or knocked down as indicated and cell lysates were analyzed by western blot assay. (b, c) PLC/PRF/5 cells stably expressing MyD88 were transfected with the indicated plasmids and were analyzed by western blot assay. (d, e) PLC/PRF/5/LV-GFP and PLC/PRF/5/LV-MyD88 cells transfected with small interfering RNA targeting Snail (siSnail) were analyzed by western blot assay and the invasive properties of the cells were analyzed by an invasion assay using Matrigel-coated Corning Chamber. Data represent mean \pm S.D. ${ }^{*}$ indicates $P<0.01$

clinical ending of HCC patients. To further identify the correlation between MyD88 and p-Akt, we first examined MyD88 expression and p-Akt levels using a western blot assay in 8 hepatoma cell lines. As shown in Figure 6a, the p-Akt levels in MyD88-low-expressing cell lines, such as Huh-7 and PLC/PRF/5, were much lower than those in 
a
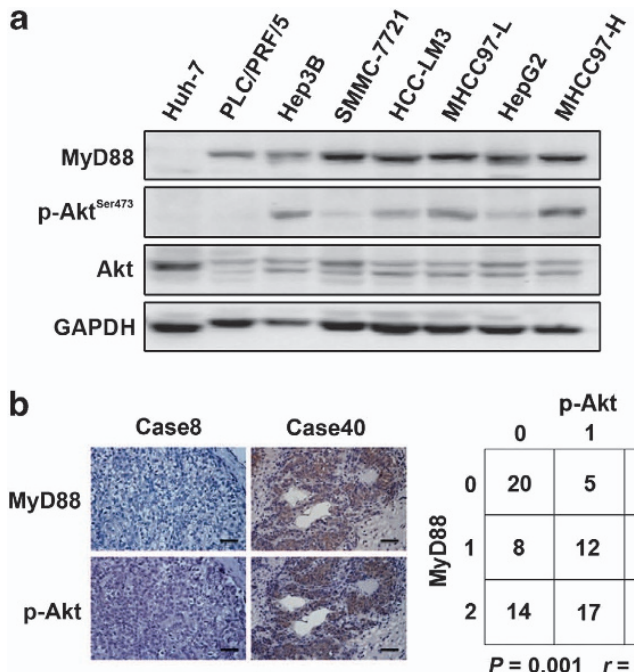

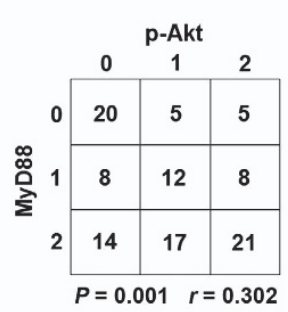

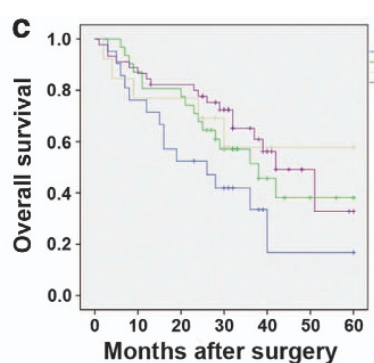

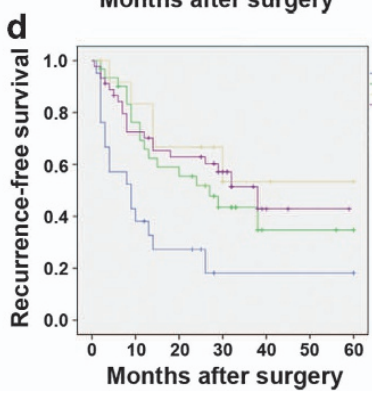

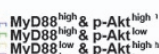

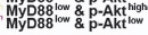
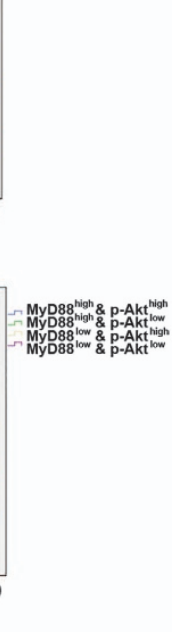

Figure 6 Combination of MyD88 and p-Akt provides better prognostic value for HCC. (a) The expression of MyD88 and the phosphorylation levels of p-Akt in hepatoma cell lines were compared by western blot assay. (b) Representative view of immunohistochemical staining of MyD88 and p-Akt and statistical correlation between MyD88 expression and p-Akt levels are shown. Scale bar, $100 \mu \mathrm{m}$. (c, d) The overall and recurrence-free survival rates of HCC patients with high or low MyD88 and p-Akt levels were compared using Kaplan-Meier analysis

MyD88-high-expressing cell lines, including HCC-LM3, MHCC97-L, MHCC97-H, and Hep3B. Next, the MyD88 expression and p-Akt levels were examined in 110 hepatocarcinoma tissues using $\mathrm{IHC}$ analysis. The results revealed a closely positive correlation between MyD88 expression and p-Akt levels $(P=0.001, r=0.302)$. Most importantly, those patients with elevated MyD88 expression and enhanced p-Akt levels displayed the worst prognosis (Figures $6 c$ and d), indicating that combination of the two parameters may have a better prognostic value.

\section{Discussion}

Recurrence and metastasis remain the most common causes of lethal outcomes in HCC after curative resection. Therefore, it is critical to uncover the mechanisms underlying HCC metastasis. However, the detailed mechanisms of hepatocarcinogenesis and metastasis remain unclear. In our preliminary studies, we found that MyD88 was highly expressed in HCCs, and that MyD88 expression was closely associated with poor prognosis in HCC patients. Thus, the contribution of MyD88 for HCC metastasis piqued our interest. Here, we demonstrated that enhanced MyD88 was able to induce EMT and cancer stem cell-like features in hepatoma cells, hence facilitating HCC metastasis. Furthermore, we clarified that MyD88 could bind to the regulatory subunit of PI3-K, p85, and activate PI3-K/Akt/GSK-3 $\beta /$ Snail pathway to induce EMT and tumor metastasis.

EMT is reportedly involved in the progression of HCC and correlates with prognosis in patients. ${ }^{32}$ As MyD88 promoted metastatic potential of HCC cells and caused morphological changes of the tumor cells, we therefore connect the biological function of MyD88 with EMT induction. EMT-related markers were detected using real-time PCR (RT-PCR), western blot analysis, and immunofluorescence assay.
Enhanced MyD88 induced characteristics of EMT in HCC cells. These results suggest that MyD88 might promote HCC metastasis, at least partially, through the induction of EMT in hepatoma cells.

As mentioned previously, cells that have undergone the process of EMT are believed to display properties of cancer stem cells, which uncovered the relationship between tumor metastasis and stemness feature. Expectedly, our results, including in vitro cancer stem cell markers testing and in vivo cancer cells inoculation, revealed that deletion of MyD88, which was able to reverse EMT process, could extremely reduce properties of cancer stem cells. Moreover, in fresh clinical samples, the mRNA levels of MyD88 were higher in $\mathrm{EpCAM}^{+}$and $\mathrm{CD}_{133^{+}}$cancer stem cell populations compared with their corresponding control groups. These results indicate metastatic cancer cells that experienced EMT process can acquire cancer stem cell features, and MyD88 may be one of the mediators in this process.

Akt is involved in many basic cellular processes including EMT. In CRC, Akt activation triggered by TNF- $\alpha$ could induce EMT, promoting CRC progression. ${ }^{33}$ The EMT induced by activated Akt involves loss of cell-cell adhesion, morphological changes, induction of cell motility, and changes in the production or distribution of specific proteins. ${ }^{28}$ In our previous studies, we found knockdown of MyD88 resulted in remarkable reduction of $\mathrm{p}$-Akt levels. Here, we further detected the influence of forced MyD88 expression on levels of p-Akt and EMT. Results showed that enforced MyD88 expression could activate Akt to induce EMT, thus promoting metastatic potential of HCC cells. Akt is a classic downstream target of PI3-K. In inflammatory cells (the murine macrophage RAW 264.7 cell line), MyD88 was found to be able to interact with p85, the regulatory subunit of $\mathrm{PI} 3-\mathrm{K}$ stimulated by LPS, and a YXXM motif within the TIR domain of MyD88 was suggested to be important for this interaction. ${ }^{34}$ Here, by performing 
coimmunoprecipitation analysis, we discovered that MyD88 interacted with p85 independent of TLR/IL-1R-mediated response, eventually increasing the catalytic activity of $\mathrm{PI}-\mathrm{K}$ and promoting HCC metastasis via PI3-K/Akt signaling.

Snail acts as an important transcriptional repressor of E-cadherin and EMT. ${ }^{35}$ GSK-3 $\beta$ that is predominantly regulated by $\mathrm{PI}-\mathrm{K} / \mathrm{Akt}$ binds and phosphorylates Snail to regulate Snail protein stability and subcellular localization. ${ }^{31}$ Through activation of PI3-K/Akt signaling, MyD88 suppressed GSK-3 $\beta$ activity, and enhanced the level of Snail subsequently, which led to the downregulation of E-cadherin and EMT in hepatoma cells.

In conclusion, MyD88 has an important role in HCC metastasis by induction of EMT via interaction with p85 and activation of Akt/GSK-3 $\beta /$ Snail pathway, and combination of MyD88 and p-Akt analysis may serve as a valuable prognostic factor for patients with HCC.

\section{Materials and Methods \\ Cells and culture conditions. PLC/PRF/5, HepG2, and Hep3B were obtained from American Type Culture Collection (ATCC, Manassas, VA, USA). MHCC97-H, MHCC97-L, and HCC-LM3 were obtained from the Liver Cancer Institute, Zhongshan Hospital, Fudan University (Shanghai, People's Republic of China). Huh-7 and SMCC-7721 were obtained from Cell Bank of Shanghai Institutes for Biological Sciences, Chinese Academy of Sciences (Shanghai, People's Republic of China). All the cell lines used in this study were cultured in Dulbecco's modified Eagle's medium (Gibco, Grand Island, NY, USA) supplemented with $10 \%$ (v/v) fetal bovine serum (Hyclone, Logan City, UT, USA) at $37^{\circ} \mathrm{C}$ in a humidified incubator containing $5 \% \mathrm{CO}_{2}$.}

Lentivirus, small interfering RNA, and plasmids. Lentiviral vectors encoding the human MyD88 gene were generated by using pLenti6/V5-TOPO vector (Invitrogen, Carlsbad, CA, USA) and were designated as LV-MyD88. The empty vector was used as a negative control and was designated as LV-GFP. Lentiviral vectors encoding short hairpin RNAs targeting MyD88 or scramble shRNA were generated by using pLenti6/BLOCKiT-DEST (Invitrogen) as previously described, ${ }^{13}$ and were designated as shMyD 88 and shNon, respectively. Two target sites were designed for knockdown of MyD88 expression. Further details are available in the Supplementary Data.

siRNA targeting specific human Snail sites (siSnail-1 and siSnail-2) and negative control siRNA were generated by GenePharma (Shanghai, PR China). Details are available in the Supplementary Data (Supplementary Table S2).

$\Delta$ p85 (Addgene, Cambridge, MA, USA; plasmid 13432), with adominant negative p85 alpha and deleted p110 alpha binding domain, disenables PI3-K to phosphorylate Akt. DN-Akt (Addgene, plasmid 9031) was built by generating a vector backbone (pcDNA3.0) with a mutation that deprived Akt of its phosphorylation ability. Ser9A-GSK-3 $\beta$ (Addgene, plasmid 14754), was similarly established by generating $\mathrm{pcDNA3} .0$ with a mutation that was a constitutively active mutant blocking phosphorylation of GSK-3 $\beta$.

RT-PCR and western blotting. Isolation of total cellular RNA was carried out by using the NucleoSpin RNAll (740955; MACHEREY-NAGEL, Düren, Germany) and the first-strand cDNA was generated using the PrimeScript RT reagent kit (DRR037A; Takara, Dalian, People's Republic of China). The cDNA sample was then measured by RT-PCR with an Applied Biosystems (Paisley, UK) 7500 Real-Time PCR System as recommended by the manufacturer. Relative mRNA levels of different target genes were calculated depended on the $\mathrm{Ct}$ values, corrected for $\beta$-actin expression, according to the equation: $2^{-\Delta \Delta \mathrm{Ct}}\left(\Delta \Delta \mathrm{C}_{\mathrm{t}}=\Delta \mathrm{C}_{\mathrm{t}}\right.$ (treatment) $-\Delta \mathrm{Ct}$ (control), $\Delta \mathrm{Ct}=\mathrm{Ct}$ (target genes) $-\mathrm{Ct}(\beta$-actin)).

Total cell lysate was prepared in $1 \times$ SDS lysis buffer $(62.5 \mathrm{mM}$ Tris- $\mathrm{HCl}, 2 \% \mathrm{w} / \mathrm{v}$ SDS, $10 \%$ glycerol, $50 \mathrm{mM}$ DTT, $0.01 \%$ w/v bromophenol blue). Proteins were separated by SDS-PAGE and transferred onto PVDF membranes $(0.45 \mu \mathrm{m}$, Millipore, Billerica, MA, USA). After probing with primary and secondary antibody, antigen-antibody complex was visualized by enhanced chemiluminescence's reagents Supersignal (Pierce, Rockford, IL, USA). The primers and antibodies used in this study are listed in the Supplementary Table S1 and Table S3, respectively.
Coimmunoprecipitation analysis. Cells were harvested in RIPA lysis buffer $(50 \mathrm{mM}$ Tris- $\mathrm{HCl}, 150 \mathrm{mM} \mathrm{NaCl}, 1 \% \mathrm{v} / \mathrm{v} \quad \mathrm{NP}-40,0.5 \%$ w/v sodium deoxycholate, $0.1 \% \mathrm{w} / \mathrm{v}$ SDS) supplemented with Mammalian Protease Inhibitor Mixture (Biocolors, Shanghai, People's Republic of China; $\times 100)$. The cell lysate was immunoprecipitated (IP) with anti-MyD88 antibody (Cell Signaling Technology, Boston, MA, USA), separated by SDS-PAGE and subjected to western blot analysis with anti-p85 antibody (Cell Signaling Technology).

PI3-Kinase activity assay. PI3-kinase of hepatoma cells was IP with anti-p85 antibody (Cell Signaling Technology) and Protein A/G PLUS-Agarose beads (Santa Cruz Biotechnology Inc, Santa Cruz, CA, USA). PI3-K activity in the immunoprecipitates was analyzed by $\mathrm{PI} 3-\mathrm{K}$ enzyme-linked immunosorbent assay kit (Echelon Biosciences, Salt Lake City, UT, USA) according to the manufacturer's instructions.

Cell migration and invasion assay. Cell migration ability was evaluated by Transwell Permeable Support (Corning, Corning, NY, USA), and cell invasion ability was assessed by Corning chamber (Transwell Permeable Support) coated with Matrigel (BD Biosciences, San Jose, CA, USA) according to the manufacturer's directory. For the Cell invasion assay, HCC cells were incubated for $48 \mathrm{~h}$ (Hep3B) or $60 \mathrm{~h}(\mathrm{PLC} / \mathrm{PRF} / 5)$; for the migration assay, cells were incubated for $24 \mathrm{~h}$ (Hep3B) or $48 \mathrm{~h}$ (PLC/PRF/5). Three 100-multiple microscopic fields were randomly selected to calculate the total count of the invaded or migrated cells. The relative number of cells having penetrated the ECM or basement membrane was used to denote the invasion or migration ability of the cells. All assays were carried out three times.

Animal studies. Male SCID mice and athymic BALB/c nude mice were purchased from the Shanghai Experimental Animal Center of Chinese Academic of Sciences (Shanghai, People's Republic of China) and were maintained in specific pathogen-free conditions. All animal experiments met the guidelines set by the National Institutes of Health. PLC/PRF/5 and Hep3B cells were suspended in $100 \mu \mathrm{L}$ of Dulbecco's modified Eagle medium and Matrigel $(1: 1)$ (BD Biosciences) and injected subcutaneously into SCID mice. The size and incidence of subcutaneous tumors were recorded. Six-week-old male BALB/c nude mice were randomized into two groups: the first group contained four nude mice, and the second contained eight. The second group was inoculated with PLC/PRF/5/ LV-MyD88 cells $\left(2 \times 10^{6}\right)$ and the first group was inoculated with control cells $\left(2 \times 10^{6}\right)$ in spleens. Two weeks after the intrasplenic injections, half of the second group were randomly given an intragastric administration of MK2206 (Selleck Chemicals, Houston, TX, USA), the inhibitor of Akt. Meanwhile, the other half of the second group and the first group were given normal saline as control. Here MK2206, or normal saline were dissolved in $0.5 \%$ sodium carboxyl methyl cellulose solution, concentrated at $20 \mu \mathrm{g} / \mathrm{ml}$. Each mouse was given $100 \mu \mathrm{l}$ solutions that had been disposed with ultrasonic concussion. All the mice were killed 5 weeks after inoculation, and the amount of metastatic tumor colonies in the liver was measured.

Patient samples and immunohistochemical staining. In all, the 110 HCC tissues and their corresponding nearby non-tumor livers utilized in this study were obtained from Guangxi Cancer Hospital immediately after surgical resection. Patient consent and approval from the Guangxi Cancer Hospital Ethics Committee were obtained in order to use these clinical materials for research purposes. The clinicopathologic characteristics of the patients and the follow-up were described previously. ${ }^{13}$ The expressions of MyD88, E-cadherin, and $p$-Akt in the specimens were detected by immunohistochemical assay. More details are available in Supplementary Materials and Materials and Methods.

SP analysis using flow cytometry. The cells were detached from the dishes with trypsin and suspended at $1 \times 10^{6} \mathrm{cell} / \mathrm{sl}$ in Dulbecco's modified Eagle's medium (Gibco) supplemented with $10 \%(\mathrm{v} / \mathrm{v})$ fetal bovine serum (Hyclone). These cells were then incubated at $37^{\circ} \mathrm{C}$ for $90 \mathrm{~min}$ with $20 \mu \mathrm{g} / \mathrm{ml}$ Hoechst 33342 (Sigma, St. Louis, MO, USA), either alone or in the presence of $50 \mu \mathrm{mol} / \mathrm{l}$ verapamil (Sigma). After incubation, $10 \mu \mathrm{g} / \mathrm{ml}$ propidium iodide (Beyotime, Nantong, People's Republic of China) was added and then filtered through a $40 \mu \mathrm{m}$ cell strainer (BD Falcon, Franklin Lakes, NJ, USA) to obtain single-suspension cells. Cell analyses were performed using BD Influx high-speed cell sorter (BD Biosciences). Hoechst 33342 was excited with the UV laser at 
$350 \mathrm{~nm}$ and fluorescence emission was measured with 460/50 (Hoechst blue) and $670 / 30$ (Hoechst red) optical filters. Propidium iodide labeling was measured through the 593/40 filter for the discrimination of dead cells.

Fresh clinical tissue specimens. Fresh human liver tumor specimens were obtained in accordance with the ethical standards of the institutional committee on human experimentation from the patient undergoing hepatectomy for HCC. The specimens were collected at Changhai Hospital of the Second Military Medical University in Shanghai. No previous local or systemic treatment had been conducted on the patients before operation. The surgical specimens were obtained at the time of resection from the patient and were received in the laboratory within $20 \mathrm{~min}$, immediately mechanically minced and digested with Type IV Collagenase (Sigma-Aldrich, St. Louis, MO, USA) for $30 \mathrm{~min}$ at $37^{\circ} \mathrm{C}$, before being suspended in Dulbecco's modified Eagle's medium (Gibco) containing penicillin $(500 \mathrm{U} / \mathrm{ml})$ and streptomycin $(500 \mathrm{mg} / \mathrm{ml})$. Single-cell suspensions were obtained by filtration through a $100 \mu \mathrm{m}$ filter (BD Biosciences). Dead cells and red blood cells were removed using the Ficoll gradient centrifugation method. Remaining red blood cells were lysed with ACK buffer (Beyotime). The number of viable cells was counted and analyzed using trypan blue staining. Afterwards, $\mathrm{EpCAM}^{+}$and $\mathrm{EpCAM}^{-}$populations, $\mathrm{CD}_{133^{+}}$and $\mathrm{CD} 133^{-}$populations were isolated by Magnetic Bead Cell Sorting. For magnetic cell sorting, cells were labeled with PE-conjugated EpCAM (eBioscience, San Diego, CA, USA) or CD133 antibody (Miltenyi Biotec, Cologne, Germany) followed by anti-PE microbeads (Miltenyi Biotec).

Immunofluorescence. The cells were washed three times with PBS after being fixed with $4 \%$ formaldehyde for $15 \mathrm{~min}$. Cells were then incubated with blocking buffer $(1.25 \mathrm{ml}$ fetal bovine serum plus $23.75 \mathrm{ml}$ PBS plus $75 \mu \mathrm{l}$ Triton-100

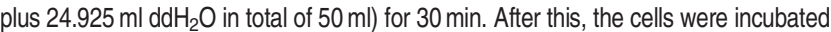
with the primary antibody (E-cadherin (Cell Signaling Technology), N-cadherin (Santa Cruz Biotechnology Inc) and vimentin (Cell Signaling Technology)) overnight at $4{ }^{\circ} \mathrm{C}$. After thorough washing, the cells were then incubated with Alexa Fluor 555 donkey anti-rabbit IgG (Life technologies, Düren, Germany). Finally, the cells were washed and mounted with DAPI (4', 6-diamidino-2-phenylindole, Dojindo Laboratories, Kumamoto, Japan) for $5 \mathrm{~min}$. Images were captured using a Leica DMIRB fluorescence microscope (OLYMPUS IX71, Tokyo, Japan).

Statistical analyses. All statistical analyses were carried out using SPSS 18.0 software (Chicago, IL, USA). Two-tailed Student's t-test was used for comparisons of two independent groups. Spearman rank correlation test was used for analysis of bivariate correlation. Kaplan-Meier analysis was used to assess the patient survival between subgroups. Overall survival was defined as the interval between surgery and death or between surgery and the last observation point. For surviving patients, the data were censored at the last follow-up. Recurrence-free survival was defined as the interval between the date of surgery and the date of diagnosis of any type of relapse (intrahepatic recurrence and extrahepatic metastasis). Data were presented as the mean \pm S.D. $P$-value $<0.05$ was considered statistically significant.

\section{Conflict of Interest}

The authors declare no conflict of interest.

Acknowledgements. This work is supported in part by grants from Ministry of Science and Technology of China '973' and '863' programs (2010CB945600, 2010CB833600, and 2011CB966200), National Nature Science Foundation of China, State Key Project for Infection Disease and New Drug Development and Programs of Shanghai Subject Chief Scientists, Municipal Commission of Education, and Municipal Commission of Science and Technology.

\section{Author contributions}

R-J Jia and $L$ Cao contributed to acquisition of data and manuscript writing L Zhang contributed to acquisition of data. W Jing, M-H Zhu, S-W Guo, G-B Wu and $\mathrm{H}$ Wang contributed to material support. $\mathrm{R}$ Chen, $X-Y$ Fan and $Y-Y$ Zhang contributed to technical support. X-Y Zhou contributed to study design and data analysis. J Zhao contributed to study design, data analysis and manuscript writing and Y-J Guo contributed to study supervision.
1. Karin M, Greten FR. NF-kappaB: linking inflammation and immunity to cancer development and progression. Nat Rev Immunol 2005; 5: 749-759.

2. Coussens LM, Werb Z. Inflammation and cancer. Nature 2002; 420: 860-867.

3. Mantovani A, Allavena P, Sica A, Balkwill F. Cancer-related inflammation. Nature 2008; 454: 436-444.

4. Rakoff-Nahoum S, Medzhitov R. Toll-like receptors and cancer. Nat Rev Cancer 2009; 9: $57-63$.

5. Naugler WE, Sakurai T, Kim S, Maeda S, Kim K, Elsharkawy AM et al. Gender disparity in liver cancer due to sex differences in MyD88-dependent IL-6 production. Science 2007; 317: 121-124.

6. Rakoff-Nahoum S, Medzhitov R. Regulation of spontaneous intestinal tumorigenesis through the adaptor protein MyD88. Science 2007; 317: 124-127.

7. Swann JB, Vesely MD, Silva A, Sharkey J, Akira S, Schreiber RD et al. Demonstration of inflammation-induced cancer and cancer immunoediting during primary tumorigenesis. Proc Natl Acad Sci USA 2008; 105: 652-656.

8. Coste I, Le Corf K, Kfoury A, Hmitou I, Druillennec S, Hainaut P et al. Dual function of MyD88 in RAS signaling and inflammation, leading to mouse and human cell transformation. J Clin Invest 2010; 120: 3663-3667.

9. Wang EL, Qian ZR, Nakasono M, Tanahashi T, Yoshimoto K, Bando $Y$ et al. High expression of Toll-like receptor $4 /$ myeloid differentiation factor 88 signals correlates with poor prognosis in colorectal cancer. Br J Cancer 2010; 102: 908-915.

10. Szajnik M, Szczepanski MJ, Czystowska M, Elishaev E, Mandapathil M, Nowak-Markwitz E et al. TLR4 signaling induced by lipopolysaccharide or paclitaxel regulates tumor survival and chemoresistance in ovarian cancer. Oncogene 2009; 28: 4353-4363.

11. Kelly MG, Alvero AB, Chen R, Silasi DA, Abrahams VM, Chan S et al. TLR-4 signaling promotes tumor growth and paclitaxel chemoresistance in ovarian cancer. Cancer Res 2006: 66: 3859-3868.

12. Roessler S, Jia HL, Budhu A, Forgues M, Ye QH, Lee JS et al. A unique metastasis gene signature enables prediction of tumor relapse in early-stage hepatocellular carcinoma patients. Cancer Res 2010; 70: 10202-10212.

13. Liang B, Chen R, Wang T, Cao L, Liu Y, Yin F et al. Myeloid differentiation factor 88 promotes growth and metastasis of human hepatocellular carcinoma. Clin Cancer Res 2013; 19: 2905-2916.

14. Lee JM, Dedhar S, Kalluri R, Thompson EW. The epithelial-mesenchymal transition: new insights in signaling, development, and disease. J Cell Biol 2006; 172: 973-981.

15. Yang J, Weinberg RA. Epithelial-mesenchymal transition: at the crossroads of development and tumor metastasis. Dev Cell 2008; 14: 818-829.

16. Mani SA, Guo W, Liao MJ, Eaton EN, Ayyanan A, Zhou AY et al. The epithelial-mesenchymal transition generates cells with properties of stem cells. Cell 2008; 133: 704-715.

17. Morel AP, Lievre M, Thomas C, Hinkal G, Ansieau S, Puisieux A. Generation of breast cancer stem cells through epithelial-mesenchymal transition. PLoS One 2008; 3: e2888.

18. Peinado H, Olmeda D, Snail Cano A. Zeb and bHLH factors in tumour progression: an alliance against the epithelial phenotype? Nat Rev Cancer 2007; 7: 415-428.

19. Sanchez-Tillo E, Liu Y, de Barrios O, Siles L, Fanlo L, Cuatrecasas M et al. EMT-activating transcription factors in cancer: beyond EMT and tumor invasiveness. Cell Mol Life Sci 2012; 69: 3429-3456.

20. Massague J. TGFbeta in cancer. Cell 2008; 134: 215-230

21. Huber MA, Kraut N, Beug H. Molecular requirements for epithelial-mesenchymal transition during tumor progression. Curr Opin Cell Biol 2005; 17: 548-558.

22. Thiery JP. Epithelial-mesenchymal transitions in tumour progression. Nat Rev Cancer 2002; 2: 442-454.

23. Zheng YW, Taniguchi H. Diversity of hepatic stem cells in the fetal and adult liver. Semin Liver Dis 2003; 23: 337-348.

24. Yamashita T, Ji J, Budhu A, Forgues M, Yang W, Wang HY et al. EpCAM-positive hepatocellular carcinoma cells are tumor-initiating cells with stem/progenitor cell features. Gastroenterology 2009; 136: 1012-1024.

25. Ma S, Chan KW, Hu L, Lee TK, Wo JY, Ng IO et al. Identification and characterization of tumorigenic liver cancer stem/progenitor cells. Gastroenterology 2007; 132: 2542-2556.

26. Yang ZF, Ho DW, Ng MN, Lau CK, Yu WC, Ngai P et al. Significance of CD90 + cancer stem cells in human liver cancer. Cancer Cell 2008; 13: 153-166.

27. Kondo $T$, Setoguchi T, Taga T. Persistence of a small subpopulation of cancer stem-like cells in the C6 glioma cell line. Proc Natl Acad Sci U S A 2004; 101: $781-786$

28. Grille SJ, Bellacosa A, Upson J, Klein-Szanto AJ, van Roy F, Lee-Kwon W et al. The protein kinase Akt induces epithelial mesenchymal transition and promotes enhanced motility and invasiveness of squamous cell carcinoma lines. Cancer Res 2003; 63: 2172-2178.

29. Larue L, Bellacosa A. Epithelial-mesenchymal transition in development and cancer: role of phosphatidylinositol 3' kinase/AKT pathways. Oncogene 2005; 24: 7443-7454.

30. Altomare DA, Testa JR. Perturbations of the AKT signaling pathway in human cancer. Oncogene 2005; 24: 7455-7464.

31. Zhou BP, Deng J, Xia W, Xu J, Li YM, Gunduz M et al. Dual regulation of Snail by GSK-3beta-mediated phosphorylation in control of epithelial-mesenchymal transition. Nat Cell Biol 2004; 6: 931-940. 
32. Yang MH, Chen CL, Chau GY, Chiou SH, Su CW, Chou TY et al. Comprehensive analysis of the independent effect of twist and snail in promoting metastasis of hepatocellular carcinoma. Hepatology 2009; 50: 1464-1474.

33. Wang $\mathrm{H}$, Wang $\mathrm{HS}$, Zhou BH, Li CL, Zhang $\mathrm{F}$, Wang XF et al. Epithelial-mesenchymal transition (EMT) induced by TNF-alpha requires AKT/GSK-3beta-mediated stabilization of snail in colorectal cancer. PLoS One 2013; 8: e56664.

34. Laird MH, Rhee SH, Perkins DJ, Medvedev AE, Piao W, Fenton MJ et al. TLR4/MyD88/PI3K interactions regulate TLR4 signaling. J Leukoc Biol 2009; 85: 966-977.
35. Cano A, Perez-Moreno MA, Rodrigo I, Locascio A, Blanco MJ, del Barrio MG et al. The transcription factor snail controls epithelial-mesenchymal transitions by repressing E-cadherin expression. Nat Cell Biol 2000; 2: 76-83.

(cc) (i) $\odot$ Cell Death and Disease is an open-access journal published by Nature Publishing Group. This work is licensed under a Creative Commons Attribution-NonCommercialNoDerivs 3.0 Unported License. To view a copy of this license, visit http://creativecommons.org/licenses/by-nc-nd/3.0/

Supplementary Information accompanies this paper on Cell Death and Disease website (http://www.nature.com/cddis) 